Review began 10/04/2021 Review ended 10/29/2021 Published 10/30/2021

(c) Copyright 2021

Li et al. This is an open access article distributed under the terms of the Creative Commons Attribution License CC-BY 4.0. which permits unrestricted use, distribution, and reproduction in any medium, provided the original author and source are credited.

\section{Characteristics Assessment of Online YouTube Videos on Radiotherapy for Lung Cancer}

\author{
Jim (Zhang Hao) $\mathrm{Li}^{1}$, Meredith Giuliani ${ }^{2}$, Paris-Ann Ingledew ${ }^{1}$ \\ 1. Department of Surgery, Division of Radiation Oncology, University of British Columbia, Faculty of Medicine, \\ Vancouver, CAN 2. Department of Radiation Oncology, University of Toronto, Toronto, CAN
}

Corresponding author: Paris-Ann Ingledew, pingledew@bccancer.bc.ca

\section{Abstract \\ Introduction}

The internet has become a mainstay source of health information for cancer patients. Online patient education videos are common; however, there have been no studies examining the quality of publicly available videos on radiotherapy for lung cancer (one of the most common forms of cancer). To fill this knowledge gap, we aim to systematically map and objectively assess videos discussing radiotherapy for lung cancer on YouTube.

\section{Methods}

The terms "radiotherapy for lung cancer," "radiation for lung cancer," "radiation therapy for lung cancer," and "radiation treatment for lung cancer" were searched on YouTube using a clear-cache browser. Results were sorted by relevance and the top 50 English-language results for each search were recorded. After removing duplicates, each video was assessed for length, Video Power Index (VPI, which is the product of a video's average daily views and like and dislike ratio), source, content, comment moderation, and misinformation. Two raters were used to ensure consistency. Results were evaluated using descriptive and inferential statistics.

\section{Results}

A total of 88 unique videos resulted from the search. The median video length was 4 minutes and 5 seconds. The average VPI was 10.9 (95\% CI: 1.5-20.4) and the median number of views was 954.5 . All videos were published between July 8, 2009 and November 18, 2020. Of the videos, 44\% were published within the past two years. A total of $61 \%$ of the videos were from the USA, 14\% were from the UK, $6 \%$ from Australia, $5 \%$ each from Canada and India, and other countries make up the remaining 10\%. Most of the videos were published by healthcare facilities (39\%) and non-profit organizations (31\%). Content-wise, 95\% of videos contain information specific to lung cancer. A total of 46 videos (52\%) were targeted toward patient education. Of which, 37 covered radiotherapy for lung cancer, 12 covered side effects for radiotherapy, and 11 covered both. The other 42 videos (48\%) were designed for a professional audience. Stereotactic body radiation therapy (SBRT)/stereotactic ablative radiotherapy (SABR) was the most commonly described radiotherapy modality (42\%), and the physician interview was the most common format, being used in 59\% of videos. Out of the 38 videos with at least one comment, only two (5\%) were moderated by the host channel. None of the videos featured misleading information.

\section{Conclusions}

This study comprehensively surveyed YouTube videos pertaining to radiotherapy for lung cancer to provide a high-level overview of the information that patients may find online. Although nearly half of the videos describe lung cancer radiotherapy for patients, only a small proportion comprehensively cover both radiotherapy and its side effects. The results of our study can help guide the development of patient education tools and encourage healthcare providers to recognize the limitations of online health information and proactively address patient questions regarding radiotherapy. Future research could examine videos on other lung cancer treatment options or radiotherapy for other cancers.

Categories: Oncology, Healthcare Technology

Keywords: online health information, youtube videos, radiotherapy (rt), lung cancer, patient education

\section{Introduction}

Lung cancer is the leading cause of cancer deaths worldwide and ranks the second-highest in incidence [1]. Currently, radiation therapy (RT) is one of the main modalities of lung cancer treatment, as it offers a survival benefit for patients at various stages of small cell lung cancer (SCLC) and non-small cell lung cancer (NSCLC) $[2,3]$. However, not all patients who are eligible receive it. It has been estimated that $64 \%$ of NSCLC patients require RT, but in practice, only $32 \%$ of NSCLC patients in the United States receive RT as part of 
their treatment $[2,4]$. This proportion is even smaller in low-income countries, where less than $10 \%$ of eligible patients receive RT [5]. Besides lack of insurance and treatment availability, other commonly cited barriers to receiving RT include lack of information and inaccurate perceptions regarding RT, its efficacy, and side effects [6-8]. Access to reliable and accurate information is therefore critical to ensuring that patients can receive RT when indicated.

To illustrate the role of online health information, $80 \%$ of internet users are seeking health information online, a trend that has been increasing in recent years $[9,10]$. In particular, YouTube is becoming more prominent as a platform for disseminating health information [11]. It is the second most visited website in the world, where users may search for medical information using its large, diverse, and free-to-access video library [12]. Although research on personal health can be empowering for patients [13], a seminal 2012 systematic review revealed concerns regarding the prevalence of misleading information and lack of content regulation on YouTube [11]. This in turn creates potentially harmful situations where patients receive conflicting information, which could result in delays or refusal of necessary interventions such as RT for lung cancer. Previous research has shown that only $19.3 \%$ of text-based websites discussing adverse events of RT for lung cancer were considered high-quality as per the Health on the Net Foundation Code of Conduct (HONcode) framework and that this trend is consistent across multiple languages [14]. Other recent studies examining the quality of YouTube videos for other cancers have generally demonstrated considerable space for improvement and concluded that YouTube is insufficient on its own to provide reliable patient education $[15,16]$. However, to the best of our knowledge, there are no specific studies examining the quality of YouTube videos regarding RT for lung cancer. This indicates a gap in the literature, which we will attempt to address through conducting this study.

\section{Materials And Methods \\ Extracting videos}

The terms "radiotherapy for lung cancer," "radiation for lung cancer," "radiation therapy for lung cancer," and "radiation treatment for lung cancer" were inputted into the YouTube search engine. The results were sorted by "Relevance," and the top 50 results for each search were recorded, with the aim of capturing all videos that patients would have reasonably been exposed to. After removing duplicates, we screened all results against pre-determined inclusion criteria: videos must be (a) currently accessible (i.e. not scheduled for future release), (b) not behind a paywall, and (c) in the English language. A rather lenient set of inclusion criteria was employed since the primary purpose of this research was to objectively characterize what resources are available to patients. To minimize undue influences from the researcher's geographical location and search history, all searches were performed using a clear-cache Google Chrome browser (Google Inc., Mountain View, CA) in incognito mode.

\section{Characteristics assessment}

To the best of our knowledge, there is no comprehensive standard pre-existing tool for assessing online videos in the patient education setting [17]. Although traditional tools exist for evaluating the quality of online patient education materials, such as the Health on the Net (HON) Foundation code [18], the DISCERN scale [19], and the Journal of American Medical Association (JAMA) benchmarks [20], they are not specifically designed for videos. As such, rather than focus on an evaluation of quality, for the purposes of this study, we developed a systematic approach to collect objective video parameters. The approach was divided into three broad domains: general parameters, video source, and video content. Within the general parameters domain, each video was assessed for its number of views, date of publication, number of likes and dislikes, Video Power Index (VPI, which is the product of a video's average daily views and like and dislike ratio) [21], and video length. It should be noted that VPI is a commonly used parameter in similar research to objectively measure video popularity by taking into account the average number of views per day and the like and dislike ratio, but it has no bearing on quality [21]. The video source domain specified each video's country of origin, publisher, and presenter(s). Finally, the video content domain included topic(s) covered, media type, comment moderation, advertisements, and gross misinformation. These parameters were selected as they correlated or were analogous to previously validated markers used to characterize health information in traditional media such as books or websites [18-20]. For example, the "publisher" and “presenter(s)" are analogous to disclosing authorship as per the JAMA benchmarks [20]. Similar approaches have been employed by other studies examining the content of online health videos - common metrics include the number of views, like and dislike ratio, video length, publisher, and topic(s) covered [22-24]. After data collection, results were evaluated using descriptive statistics (i.e., measures of central tendency) and inferential statistics (i.e., chi-square test) using the statistical software R, version 3.6.1 (R Foundation, Vienna, Austria).

\section{Inter-rater reliability}

The reliability of the video descriptions was confirmed via consensus between two independent assessments by a radiation oncologist with lung cancer expertise and a medical student. A number was assigned to each video and a random number generator was used to select a random sample of 10 videos for two researchers to evaluate independently by applying the characterization approach. Inter-rater reliability was highly consistent among both raters; for every category, a kappa value or intraclass correlation coefficient $>0.70$ 


\section{Cureus}

was achieved, thereby indicating good inter-rater agreement. Any discrepancies were resolved by discussion and the remaining videos were then assessed by one researcher independently.

\section{Results}

\section{General parameters}

The top 50 YouTube results for the search terms "radiotherapy for lung cancer," "radiation for lung cancer," "radiation therapy for lung cancer," and "radiation treatment for lung cancer” were compiled. After applying the exclusion criteria and removing all duplicates, 88 unique videos remained.

The popularity of YouTube videos covering radiotherapy for lung cancer followed a positively skewed distribution. Although the mean number of views per video was 25,487 , the median was only 955 . The range varied from 46 to $1,228,659$ views. It should be noted that the video with 1,228,659 views received more views than all the other 87 videos combined. The newest video was published 52 days before the time of the search, while the oldest was already published for 4,203 days. A total of $69 \%$ of videos were more than two years old and 57\% were more than three years old. The Video Power Index (VPI) was calculated by multiplying a video's average daily views by its like and dislike ratio [21]. Similar to the number of views, the VPI also followed a positively skewed distribution, with a mean of 10.9 and a median of 1.0 (Figure 1). VPI ranged from 0.05 to 376 . The median video length was 4 minutes and 5 seconds. Videos from commercial channels had a median length of 2 minutes and 11 seconds while those from non-commercial channels had a median length of 4 minutes and 32 seconds.

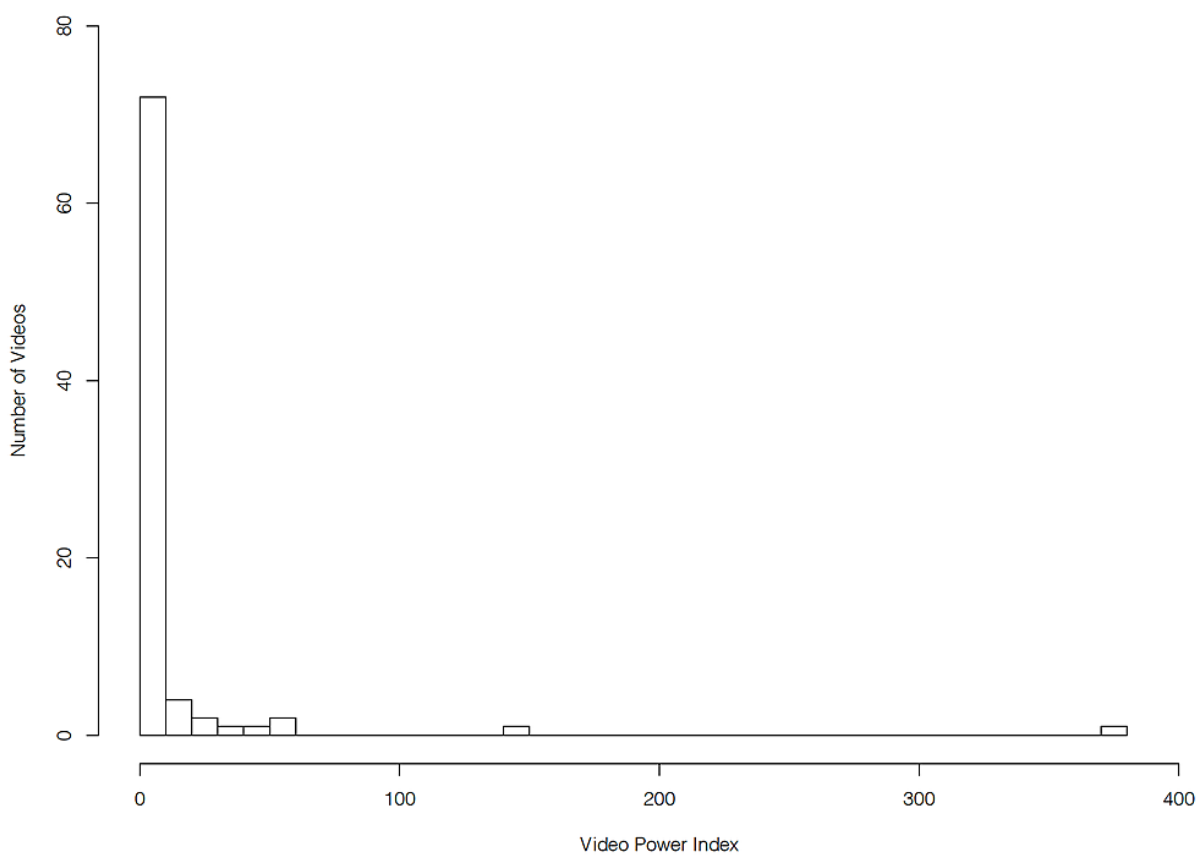

FIGURE 1: Histogram of the Video Power Index of YouTube videos describing radiotherapy for lung cancer.

\section{Video source}

Most videos originated from the USA (61\%), followed by the UK (14\%), Australia (6\%), Canada (5\%), and India (5\%). The remaining $10 \%$ were from other countries (Table 1 ). The publisher channels were categorized into healthcare, non-profits, commercial, education, and personal. Most videos were from either a healthcare (39\%) or non-profit (31\%) publisher. A total of $18 \%$ were from commercial sources, $9 \%$ from educational channels, and $3 \%$ from personal accounts (Table 1). Information was presented by a physician and/or allied health workers in $77 \%$ of videos, while information was presented by a patient in $18 \%$ of videos. Non-profit publishers were more likely to feature a patient presenter compared to other publisher categories $(\mathrm{P}=0.014)$. 


\section{Cureus}

\begin{tabular}{|c|c|c|}
\hline Parameter & Results & Number of videos (percent of total) \\
\hline \multirow{3}{*}{ Date of video publication } & $<2$ years ago & $27(31)$ \\
\hline & $2-3$ years ago & $11(12)$ \\
\hline & $>3$ years ago & $50(57)$ \\
\hline \multirow{8}{*}{ Video source } & USA & $54(61)$ \\
\hline & UK & $12(14)$ \\
\hline & Australia & $5(6)$ \\
\hline & Canada & $4(5)$ \\
\hline & India & $4(5)$ \\
\hline & France & $2(2)$ \\
\hline & The Netherlands & $2(2)$ \\
\hline & Other countries & $5(6)$ \\
\hline \multirow{5}{*}{ Publisher affiliation } & Healthcare facility & $34(39)$ \\
\hline & Non-profit & $27(31)$ \\
\hline & Commercial & $16(18)$ \\
\hline & Educational institution & $8(9)$ \\
\hline & Personal & $3(3)$ \\
\hline \multirow{5}{*}{ Media type/video format* } & Physician interview & $52(59)$ \\
\hline & Video tours & $20(23)$ \\
\hline & Patient interviews & $19(22)$ \\
\hline & Lecture-style presentations & $17(19)$ \\
\hline & Computer graphics & $11(13)$ \\
\hline \multirow{3}{*}{ Radiotherapy modality* } & Stereotactic body radiation therapy & $37(42)$ \\
\hline & Intensity-modulated radiation therapy & $11(13)$ \\
\hline & Proton therapy & $9(10)$ \\
\hline \multirow{4}{*}{ Comment moderation } & Present & $2(2)$ \\
\hline & Absent & $36(41)$ \\
\hline & No comments seen & $30(34)$ \\
\hline & Comments disabled & $20(23)$ \\
\hline
\end{tabular}

\section{TABLE 1: Compiled categorical results of YouTube videos on radiotherapy for lung cancer.}

* Resultant categorical variables not mutually exclusive with each other.

\section{Video content}

Of the 88 included videos, 46 (52\%) were subjectively assessed to be targeted toward a patient audience. The other 42 (48\%) were designed for health professionals. Although 84 videos (95\%) presented information related to lung cancer, only 52 described radiation therapy (RT) for its treatment, and 18 covered the side effects of RT. In total, 11 videos (13\%) were directed toward a patient audience while adequately describing RT for lung cancer and its side effects (Figure 2). 


\section{Cureus}

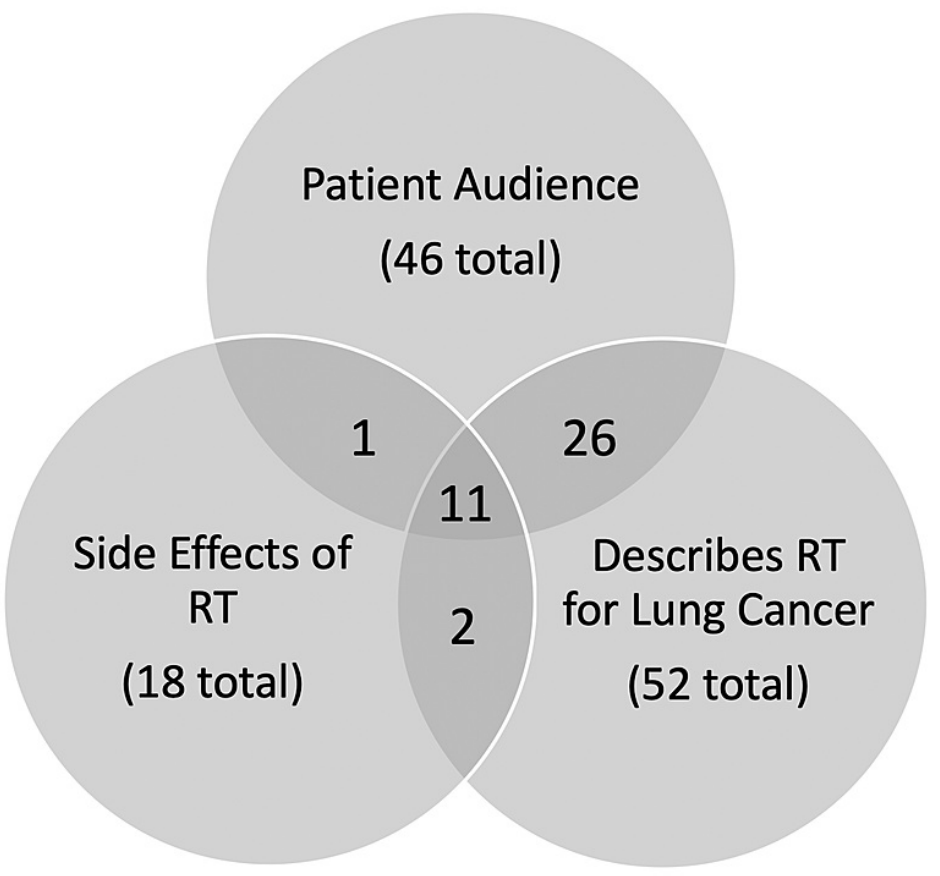

FIGURE 2: Venn diagram of the number of videos intended for patient education, describing radiotherapy for lung cancer, and explaining the side effects of radiotherapy.

$\mathrm{RT}=$ radiation therapy

The physician interview was the most commonly used format, being featured in $59 \%$ of videos, followed by live video tours (23\%), patient interviews (22\%), lecture-style presentations (19\%), and computer graphics/animations (13\%) (Table 1). Some videos employed multiple formats. In terms of RT modalities, as previously mentioned, only 52 (59\%) videos described at least one modality. Stereotactic body radiation therapy (SBRT) was mentioned by 37 videos ( $42 \%$ of total), followed by intensity-modulated radiation therapy (IMRT) at 13\% and proton therapy at 10\% (Table 1). Again, multiple modalities could be described in a single video. Comments were enabled in 68 videos. Of these, 38 had at least one comment. However, only two videos' comment sections were moderated by the publisher, as evidenced by them responding to at least one viewer comment (Table 1). Advertisements were seen in six videos (7\%). None of the videos featured grossly inaccurate or misleading information.

\section{Discussion}

Out of the 88 videos selected for characterization, only 27 were published within the past two years, and the majority (50 videos) were published more than three years ago. This raises a point of concern as patients may not necessarily be receiving up-to-date information about treatment options. This is consistent with existing literature that suggests inadequate currency among online cancer information [25]. We acknowledge that our search was filtered by "Relevance" instead of "Upload Date," where the latter would show the newest videos on top. Therefore, currency rates may be higher had we performed the search by "Upload Date." However, since "Relevance" is the default setting, we believe using this would be most representative of what patients would practically encounter. Nevertheless, our findings still indicate that YouTube alone is inconsistent in delivering up-to-date information and that healthcare professionals will need to continue informing patients about the standard of care.

Videos offer an advantage over traditional text-based education materials as they are less prone to poor readability hindering patient comprehension, and there is evidence to suggest superior knowledge retention [26]. To illustrate the effect that video format has on quality, a 2019 study on thyroid cancer videos showed that animated videos were not statistically different from non-animated videos in terms of length, VPI, and quality [15], while a more recent study by Kuru and Erken in 2020 suggested that quality may be higher in videos presented by physicians as opposed to non-physicians [27]. In the context of our study, it is encouraging that the physician interview was the most commonly used format, and we would further encourage physicians to guide patients to videos produced by other healthcare professionals. 
Commercialization of online health information is associated with lower information quality and a higher risk of featuring biased information [28]. However, as none of the videos in our study featured grossly inaccurate or misleading information, there is insufficient statistical power for us to compare between videos from commercial versus non-commercial sources. Interestingly, previous literature has suggested that objective and subjective measures of video quality are positively correlated to video length [15,23,24]. Combined with our study findings that the median length of commercial videos was shorter than their noncommercial counterparts, it is possible that a discrepancy in quality favoring non-commercial videos would exist as well. However, as our goal was not to assess video quality, this remains speculative.

With regards to the target audience, there is roughly an even distribution of videos targeted toward patients and healthcare professionals (46 versus 42). Although the "signal-to-noise" ratio may seem poor at a glance, it is reasonable to assume that most patients would skim the titles and thumbnails of the first few videos to find a video appropriate for them. A greater concern lies within video content - out of the 46 videos targeted toward a patient audience, only 11 described both what radiotherapy is and its side effects when used for cancer treatment (Figure 2). Since side effects of treatment is a topic of high patient interest [29], many patients may be left with insufficient information to guide their decision-making. Therefore, it is imperative that healthcare professionals take the time to specifically discuss details such as side effects and direct patients to appropriate resources.

Another interesting finding is the lack of visible comment moderation on the vast majority of included videos. Out of the 38 videos with at least one comment, only two comment sections were moderated by the host channel via the presence of replies. We acknowledge that some channels may moderate comments by deleting undesired content. However, it is not possible for the researchers to determine if any comments have been deleted, therefore host replies were used as a proxy. Another 30 videos had no comments. Considering the low rate of comment moderation in the videos with comments, it is unlikely that a large portion of those 30 videos would have moderated comment sections. The remaining 20 videos disabled comments altogether. The near-complete absence of comment moderation means that misinformation either intentional or unintentional - could be easily spread among viewers even if the video itself does not. This is especially pertinent for videos with a high view count. Although disabling comments may seemingly prevent the spread of misinformation, it also precludes constructive viewer interactions. Previous research has shown that comment sections can serve as a platform for collaborative deliberation, which in turn stimulates higher levels of knowledge construction among readers [30]. Especially for sensitive topics such as lung cancer, having a safe platform where patients can share their experiences, support each other, and offer constructive feedback to the channel can be of immense benefit.

Limitations to our study include the fact that it only represents a snapshot in time. The YouTube algorithm for displaying videos may change over time, and videos' view counts will increase at different rates, which further alter their visibility. In addition, due to resource constraints, our search was limited to the English language. In the future, analyses may be performed for searches in additional languages, and video characteristics may be compared to determine if a difference exists between videos in various languages. Most importantly, as an objective characteristics assessment, we did not attempt to assess videos for quality. A direction for future research would be to analyze video quality for multiple cancers and to compare them against each other.

\section{Conclusions}

Most YouTube videos describing radiotherapy for lung cancer are delivered by physicians and published by non-commercial sources. However, the currency is inconsistent, comment sections are poorly moderated, and nearly half of the videos are not targeted toward a patient audience. In addition, only a small proportion of the videos aimed toward a patient audience comprehensively cover both radiotherapy and its side effects. Nevertheless, despite the limitations of YouTube videos describing radiotherapy for lung cancer, healthcare professionals should recognize that online videos could still be a powerful form of patient education and empowerment. Not only does it circumvent traditional barriers such as reading comprehension, but it also offers the opportunity to deliver high-quality information in an era where telemedicine is becoming increasingly prominent. As it stands, there is a vast amount of unfulfilled potential that could be better harnessed to complement the patient-physician relationship in advancing patient-centered decisionmaking.

\section{Additional Information \\ Disclosures}

Human subjects: All authors have confirmed that this study did not involve human participants or tissue. Animal subjects: All authors have confirmed that this study did not involve animal subjects or tissue. Conflicts of interest: In compliance with the ICMJE uniform disclosure form, all authors declare the following: Payment/services info: All authors have declared that no financial support was received from any organization for the submitted work. Financial relationships: All authors have declared that they have no financial relationships at present or within the previous three years with any organizations that might have an interest in the submitted work. Other relationships: All authors have declared that there are no other relationships or activities that could appear to have influenced the submitted work. 


\section{References}

1. Sung H, Ferlay J, Siegel RL, Laversanne M, Soerjomataram I, Jemal A, Bray F: Global cancer statistics 2020: GLOBOCAN estimates of incidence and mortality worldwide for 36 cancers in 185 countries. CA Cancer J Clin. 2021, 71:209-49. 10.3322/caac.21660

2. Cheng M, Jolly S, Quarshie WO, Kapadia N, Vigneau FD, Kong FS: Modern radiation further improves survival in non-small cell lung cancer: an analysis of 288,670 patients. J Cancer. 2019, 10:168-77. $10.7150 /$ jca. 26600

3. Pezzi TA, Schwartz DL, Mohamed AS, et al.: Barriers to combined-modality therapy for limited-stage small cell lung cancer. JAMA Oncol. 2018, 4:e174504. 10.1001/jamaoncol.2017.4504

4. Tyldesley S, Boyd C, Schulze K, Walker H, Mackillop WJ: Estimating the need for radiotherapy for lung cancer: an evidence-based, epidemiologic approach. Int J Radiat Oncol Biol Phys. 2001, 49:973-85. 10.1016/S0360-3016(00)01401-2

5. Zubizarreta EH, Fidarova E, Healy B, Rosenblatt E: Need for radiotherapy in low and middle income countries - the silent crisis continues. Clin Oncol (R Coll Radiol). 2015, 27:107-14.

10.1016/j.clon.2014.10.006

6. Chierchini S, Ingrosso G, Saldi S, Stracci F, Aristei C: Physician and patient barriers to radiotherapy service access: treatment referral implications. Cancer Manag Res. 2019, 11:8829-33. 10.2147/CMAR.S168941

7. Sundaresan P, King M, Stockler M, Costa D, Milross C: Barriers to radiotherapy utilization: consumer perceptions of issues influencing radiotherapy-related decisions. Asia Pac J Clin Oncol. 2017, 13:e489-96. 10.1111/ajco.12579

8. Sundaresan P, King MT, Stockler MR, Costa DS, Milross CG: Barriers to radiotherapy utilisation in New South Wales Australia: health professionals' perceptions of impacting factors. J Med Imaging Radiat Oncol. 2015, 59:535-41. 10.1111/1754-9485.12334

9. Prestin A, Vieux SN, Chou WY: Is online health activity alive and well or flatlining? Findings from 10 years of the Health Information National Trends Survey. J Health Commun. 2015, 20:790-8. 10.1080/10810730.2015.1018590

10. Health information is a popular pursuit online . (2011). Accessed: April 29, 2021: https://www.pewresearch.org/internet/2011/02/01/health-information-is-a-popular-pursuit-online/.

11. Madathil KC, Rivera-Rodriguez AJ, Greenstein JS, Gramopadhye AK: Healthcare information on YouTube: a systematic review. Health Informatics J. 2015, 21:173-94. 10.1177/1460458213512220

12. The top 500 sites on the web . (2011). Accessed: June 28, 2021: https://www.alexa.com/topsites.

13. McMullan M: Patients using the Internet to obtain health information: how this affects the patient-health professional relationship. Patient Educ Couns. 2006, 63:24-8. 10.1016/j.pec.2005.10.006

14. Dee EC, Lee G: Adverse effects of radiotherapy and chemotherapy for common malignancies: what is the quality of information patients are finding online?. J Cancer Educ. 2021, 36:178-88. 10.1007/s13187-01901614-2

15. Aydin MA, Akyol H: Quality of information available on YouTube videos pertaining to thyroid cancer . J Cancer Educ. 2020, 35:599-605. 10.1007/s13187-019-01502-9

16. Loeb S, Sengupta S, Butaney M, et al.: Dissemination of misinformative and biased information about prostate cancer on YouTube. Eur Urol. 2019, 75:564-7. 10.1016/j.eururo.2018.10.056

17. Drozd B, Couvillon E, Suarez A: Medical YouTube videos and methods of evaluation: literature review. JMIR Med Educ. 2018, 4:e3. 10.2196/mededu.8527

18. Discover the 8 principles of the HONcode in 35 languages . (2019). Accessed: January 13, 2021: https://www.hon.ch/cgi-bin/HONcode/principles.pl?English.

19. The DISCERN handbook. (1998). Accessed: January 13, 2021: http://www.discern.org.uk/discern.pdf.

20. Silberg WM, Lundberg GD, Musacchio RA: Assessing, controlling, and assuring the quality of medical information on the internet: caveant lector et viewor-let the reader and viewer beware. JAMA. 1997, 277:1244-5. 10.1001/jama.1997.03540390074039

21. Erdem MN, Karaca S: Evaluating the accuracy and quality of the information in kyphosis videos shared on YouTube. Spine (Phila Pa 1976). 2018, 43:E1334-9. 10.1097/BRS.0000000000002691

22. Sampson M, Cumber J, Li C, Pound CM, Fuller A, Harrison D: A systematic review of methods for studying consumer health YouTube videos, with implications for systematic reviews. PeerJ. 2013, 1:e147. 10.7717/peerj.147

23. Sahin AN, Sahin AS, Schwenter F, Sebajang H: YouTube videos as a source of information on colorectal cancer: what do our patients learn?. J Cancer Educ. 2019, 34:1160-6. 10.1007/s13187-018-1422-9

24. Steinberg PL, Wason S, Stern JM, Deters L, Kowal B, Seigne J: YouTube as source of prostate cancer information. Urology. 2010, 75:619-22. 10.1016/j.urology.2008.07.059

25. Wasserman M, Baxter NN, Rosen B, Burnstein M, Halverson AL: Systematic review of internet patient information on colorectal cancer surgery. Dis Colon Rectum. 2014, 57:64-9. 10.1097/DCR.0000000000000011

26. Lim SL, Yang JC, Ehrisman J, Havrilesky LJ, Reed SD: Are videos or text better for describing attributes in stated-preference surveys?. Patient. 2020, 13:401-8. 10.1007/s40271-020-00416-9

27. Kuru T, Erken HY: Evaluation of the quality and reliability of YouTube videos on rotator cuff tears . Cureus. 2020, 12:e6852. 10.7759/cureus.6852

28. Storino A, Castillo-Angeles M, Watkins AA, et al.: Assessing the accuracy and readability of online health information for patients With pancreatic cancer. JAMA Surg. 2016, 151:831-7. 10.1001/jamasurg.2016.0730

29. Okuhara T, Ishikawa H, Urakubo A, Hayakawa M, Yamaki C, Takayama T, Kiuchi T: Cancer information needs according to cancer type: a content analysis of data from Japan's largest cancer information website. Prev Med Rep. 2018, 12:245-52. 10.1016/j.pmedr.2018.10.014

30. Dubovi I, Tabak I: An empirical analysis of knowledge co-construction in YouTube comments . Comput Educ. 2020, 156:103939. 10.1016/j.compedu.2020.103939 